\title{
Actividad científica e investigadora y grado de satisfacción de residentes de Cirugía Ortopédica y Traumatología en diferentes hospitales de la red sanitaria en España
}

\section{Scientific and research activity and degree of satisfaction of residents of Orthopedic Surgery and Traumatology in different hospitals of the health network in Spain}

\author{
Sanz-Reig J,* Mas-Martínez JJ,* Marín-Peña $\mathrm{O}^{\ddagger}$ \\ Clínica HLA Vistahermosa, Alicante España.
}

RESUMEN. Introducción: La presión asistencial ha provocado un desequilibrio hacia la vertiente clínica con respecto a la científica. El propósito del trabajo fue analizar la actividad asistencial e investigadora y el grado de satisfacción en los residentes de cirugía ortopédica y traumatología, en diferentes hospitales de la red sanitaria en España. Material y métodos: Encuesta de ámbito estatal realizada en el período del 25/06/2020 al 31/07/2020. Se analizaron variables demográficas y del servicio, de labores asistencial e investigadora. Se determinó la satisfacción del residente en escala continua de cero a 5. Resultados: 120 médicos haciendo residencia respondieron a la encuesta, rondando una edad media de 27 años y predominio de varones en $58 \%$. La distribución por año de dichos residentes fue de 26 R5 (21.7\%), 24 R4 (20.0\%), 45 R3 (37.5\%), 25 R2 (20.9\%). Sólo $37.5 \%$ indicaron carecer de labor de tipo asistencial asignada el día siguiente de la guardia y $45 \%$ respondieron que realizaban algún papel asistencial fuera del horario laboral del hospital. De esos interrogados, 56.7\% contestaron que su plan individual de formación no contemplaba la praxis científica ajustada a su año de residencia como un objetivo a cumplir. Ninguno de ellos tenía tiempo asignado con periodicidad semanal para acciones de investigación. El valor medio del nivel de satisfacción de estos médicos fue de 2.4 puntos. Conclusiones: La actividad asistencial
ABSTRACT. Introduction: Healthcare pressure has changed the acquisition of knowledge during residency training. The aim of this study was to analyze the clinical and research training, and level of satisfaction, during orthopedic and traumatology residency in different hospitals of the Spanish National Health Service. Material and methods: An online survey was distributed between 06/25/2020 and 07/31/2020. Demographic, institution, clinical and research variables were analyzed. Satisfaction was graded in a scale from 0 to 5 . Results: 120 residents answered the survey, having a mean age of 27 years and being 58\% male. Distribution of residents by year was 26 R5 (21.7\%), 24 R4 (20.0\%), 45 R3 (37.5\%), 25 R2 (20.9\%). Only $37.5 \%$ answered that they did not have any clinical activity the next day after medical guard, and $45 \%$ answered that they performed clinical activity outside of working hours. A total of $56.7 \%$ answered that their orthopedic training program did not include research training. No resident had specific time assigned to research activities. Mean satisfaction value was 2.4 points. Conclusions: Clinical activity shows aspects incompatible with current legislation. Orthopedic programs need improvements with regard to research training. A significant percentage of residents are unsatisfied with their training.

\section{Nivel de evidencia: IV}

* Traumatología Vistahermosa. Clínica HLA Vistahermosa.

‡ Cirugía Ortopédica y Traumatología. Hospital Universitario Infanta Leonor.

Correspondencia:

Javier Sanz-Reig

Edificio V76, Avenida Denia 76, 03013, Alicante.

E-mail: javisanz@coma.es

Recibido: 09-12-2020. Aceptado: 21-02-2021.

Citar como: Sanz-Reig J, Mas-Martínez JJ, Marín-Peña O. Actividad científica e investigadora y grado de satisfacción de residentes de Cirugía Ortopédica y Traumatología en diferentes hospitales de la red sanitaria en España. Acta Ortop Mex. 2021 35(1): 3-10. https://dx.doi.org/10.35366/100923 
presenta aspectos incompatibles con la legislación actual. Los planes individuales de adiestramiento precisan mejoras con respecto a la ocupación investigadora. Un porcentaje no despreciable de estos estudiantes de posgrado no estaban satisfechos con su plan personal de especialización.

Palabras clave: Cirugía ortopédica, residente, docencia, investigación.

\section{Introducción}

El programa de formación médica especializada mediante el sistema de residencia, conocido como sistema MIR, tiene como objetivo la adquisición por parte del residente de conocimientos, habilidades y hábitos, que le permitan desarrollar su actividad profesional en el futuro. Dicha educación se compone de aspectos clínicos, docentes, investigadores y éticos. ${ }^{1,2,3}$ La creciente presión asistencial sanitaria ha producido un desequilibrio con claro predominio de la vertiente clínica. El número creciente de horas semanales trabajadas en jornada ordinaria y complementaria favorece la aparición de problemas emocionales y conduce a la desmotivación de los médicos, incluyendo el riesgo de desencadenar un síndrome de burnout, lo cual repercutiría en su adiestramiento. ${ }^{4,5}$ No adquirir la instrucción adecuada en algún aspecto incrementa exponencialmente la probabilidad de no realizar dicha labor en el futuro. ${ }^{6}$

La educación investigadora permite ser crítico frente a la avalancha de información científica a la que se tiene acceso hoy en día, analizar estos resultados, gestionar recursos sanitarios y mejorar la calidad asistencial. Se considera una parte integral de cualquier programa de instrucción médica. ${ }^{7}$ Existen estudios sobre la construcción investigadora en los residentes de cirugía ortopédica y traumatología (COT) publicados en la literatura. ${ }^{4,8,9}$ Sin embargo, hasta donde los autores de este manuscrito conocen, no se han publicado estudios en España sobre la formación y satisfacción de dichos alumnos de posgrado de esa especialidad.

El objetivo del trabajo fue analizar la actividad asistencial e investigadora y el grado de satisfacción en quienes cursan la residencia de COT, en diferentes hospitales de la red sanitaria en España.

\section{Material y métodos}

Se diseñó un cuestionario anónimo de 30 preguntas en formularios de Google para determinar la ocupación asistencial e investigadora y el grado de satisfacción en los residentes de COT, de diferentes hospitales de la red sanitaria en España (Anexo 1). La encuesta se remitió a través de grupos de WhatsApp del 25/06/2020 al 31/07/2020.

Las variables analizadas fueron demográficas (edad, sexo), zona geográfica (provincia), características del servicio (número de adjuntos, incluyendo jefe de servicio y
Keywords: Orthopedic surgery, resident, teaching, research.

jefes de sección, cantidad de médicos, tipo de financiación del hospital). El residente tenía que indicar su año de residencia, suma de horas semanales de trabajo, conjunto de guardias realizadas al mes sin contar el período vacacional, labor asistencial asignada al día siguiente de la guardia y si realizaba alguna tarea asistencial extra fuera del horario laboral. Se le preguntaba si su plan individual de educación contemplaba como meta la práctica científica ajustada a cada año de residencia y si tenía tiempo consagrado semanalmente para tareas de investigación. A fin de determinar el nivel de actividad científica se registraron la cuantía de cursos y congresos asistidos, comunicaciones presentadas y trabajos científicos publicados en cada año de educación durante la residencia. También se asentó la situación actual con respecto al inicio del máster de formación e investigación, tema de desarrollo de la tesis doctoral e inicio de ésta. La satisfacción del residente en relación con el adiestramiento en el servicio se determinó en una escala continua de cero (nada satisfecho) a 5 (muy satisfecho).

Se investigó si el médico había realizado, o tenía planeado realizar, una rotación externa específica en un centro hospitalario nacional o internacional, y si tenía preferencia por algún campo específico al cual dedicarse tras terminar su residencia.

Dada la situación sanitaria del país en el momento de realizar la encuesta, se añadieron preguntas en torno a las perspectivas laborales a corto y medio plazo y sobre la influencia de la pandemia de coronavirus en el escenario laboral actual.

Se realizó estadística descriptiva de las variables aprovechando el programa SPSS v.20 (SPSS Inc. Chicago, USA). Se determinó la normalidad de las variables cuantitativas mediante la prueba de Kolmogorov-Smirnov. Las variables cuantitativas se describieron utilizando la media, desviación estándar, el rango e intervalo de confianza al 95\%; y las cualitativas con porcentajes. Con el objetivo de analizar las relaciones entre variables cuantitativas y cualitativas se utilizó la prueba t de Student y ANOVA y para relaciones entre variables cualitativas la prueba de $\chi^{2}$. Se consideraron significativos valores de $\mathrm{p}<0.05$.

\section{Resultados}

Durante el período de estudio, 120 médicos en residencia respondieron a la encuesta. Por localización geográfi- 


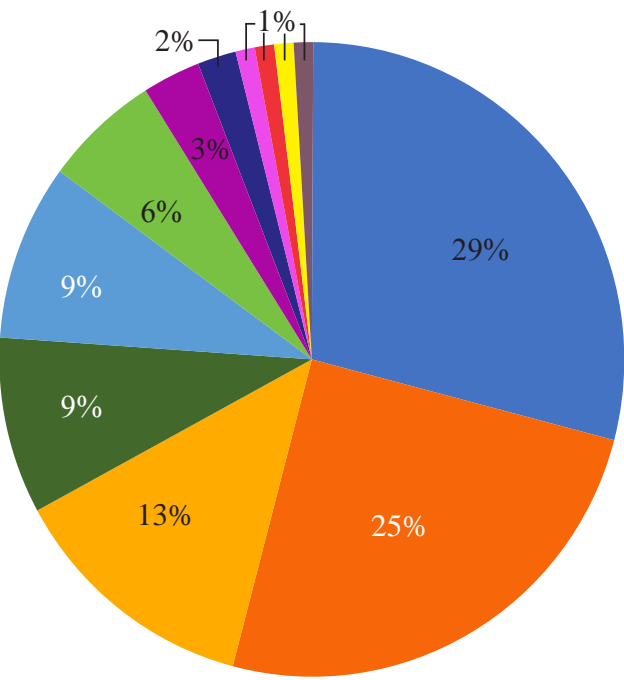

Madrid

Andalucía

Cataluña

Comunidad Valenciana

País Vasco

Murcia

Figura 1: Distribución geográfica de residentes de Cirugía Ortopédica y Traumatología. La mayoría de los médicos que respondieron a la encuesta trabajaban en la Comunidad de Madrid y la Comunidad de Andalucía.

ca, 36 de ellos (29\%) trabajaban en la Comunidad de Madrid, seguido de 31 (25\%) en la Comunidad de Andalucía (Figura 1).

La edad media de los encuestados fue de 27.7 años (DE 1.8; rango 25 a 36; IC 95\% 26.1-34.8). Por sexos, se reveló el predominio de varones en 70 casos $(58.3 \%)$ frente a mujeres en 50 casos $(41.7 \%)$.

La distribución por año de esos residentes fue de 26 R5 (21.7\%), 24 R4 (20.0\%), 45 R3 (37.5\%) y 25 R2 (20.9\%). Estos galenos trabajaban en hospitales públicos en $90 \%$ (108 casos), seguidos por hospitales públicos de gestión privada en $6.7 \%$ (ocho casos) y por hospitales privados en $3.3 \%$ (cuatro casos). El volumen de adjuntos del servicio era menor a 20 en 31 hospitales $(25.8 \%)$, entre 20 y 39 en 58 hospitales $(48.3 \%$ ) y mayor o igual a 40 en 31 hospitales (25.8\%).

Por el año de formación, el número de médicos de primero era (cinco o menos) en 32 servicios (26.7\%), segundo año (entre seis y 10) en 47 servicios (39.2\%), tercero (entre 11 y 15 ) en 24 servicios (20\%), cuarto (entre 16 y 20 ) en ocho servicios (6.7\%) y quinto (entre 21 y 25 ) en nueve servicios (7.5\%). La cantidad media de horas semanales trabajadas por el cirujano era de 37.9 (DE 3.9; rango 30 a 50; IC 95\% 32.1-48.2), con 50\% de los residentes sumando más de 38 horas de trabajo semanal. La media de guardias al mes era de 5.6 (DE 0.6; rango 4 a 7; IC 95\%: 4.2-6.7). Al mes, 4.2\% realizaban cuatro guardias, $5(35.8 \%), 6(51.7 \%)$ y 7 (8.3\%). Sólo $37.5 \%$ indicaron que no tenían labor asistencial asignada el día siguiente de la guardia, 25\% realizaba por debajo de $10 \%$ de actividad, $10.8 \%$ realizaba más de $50 \%, 8.3 \%$ realizaba más de $80 \%$ y $18.3 \%$ realizaba trabajo como una jornada habitual. De quienes respondieron, 45\% realizaban un papel asistencial fuera del horario laboral del hospital.

De dichos individuos, $56.7 \%$ contestaron que su plan personal de educación no contemplaba la praxis científica ajustada a su año de residencia como un objetivo a cumplir. Ningún médico tenía tiempo asignado con periodicidad semanal para quehaceres de investigación. El número medio ideal de horas indicado por los respondientes, que se deberían dedicar semanalmente a tareas de investigación, era de 5.7 (DE 3.1; rango 1 a 20; IC 95\% 4.8-17.6).

De tales entrevistados, $35.8 \%$ respondieron que sí existía en su servicio una motivación por parte de sus adjuntos hacia la actividad investigadora. Dicha motivación no tenía relación significativa con el volumen de residentes $(p=0.19)$, ni con el número de adjuntos del servicio $(\mathrm{p}=0.10)$.

El valor medio del nivel de satisfacción de esos médicos fue de 2.4 puntos (DE 1.0; rango 1 a 5 ; IC 95\% 1.8 4.7). Entre los encuestados, 52.5\% consideraron una puntuación igual o menor a 2 (Tabla 1). No hubo diferencias significativas en el valor medio de satisfacción al analizar el resultado por sexo $(\mathrm{p}=0.26)$, año de residencia $(\mathrm{p}=$ $0.22)$, proporción de participantes del servicio $(p=0.38)$, cantidad de adjuntos $(\mathrm{p}=0.31)$ del servicio, horas trabajadas cada semana $(\mathrm{p}=0.65)$, número de guardias mensuales $(\mathrm{p}=0.87)$ o tener carga asistencial fuera del hospital ( $\mathrm{p}$ $=0.12$ ). Aquellos médicos quienes tenían asignada labor asistencial tras la guardia tenían un valor medio de satisfacción significativamente menor que aquellos quienes no lo tenían (2.23 frente a 2.76; $\mathrm{p}=0.008)$.

Los residentes referían acudir a cursos y congresos desde el primer año de formación en un elevado porcentaje (Figura 2), así como en la elaboración y presentación de comunicaciones (Figura 3). Respecto a la realización de trabajos científicos, el volumen de cirujanos con trabajos científicos se incrementaba conforme avanzaba el tiempo de residencia, aunque en un menor porcentaje que la asistencia a cursos y congresos o la presentación de comunicaciones (Figura 4).

Sólo 9.2\% de ellos refería que había iniciado el máster de formación e investigación. Sin embargo, 30\% tenían en

Tabla 1: Nivel de satisfacción de los residentes en escala de 0 a 5.

\begin{tabular}{lccccc} 
Nivel satisfacción & 1 & 2 & 3 & 4 & 5 \\
Porcentaje & 23.3 & 29.2 & 32.5 & 11.7 & 3.3 \\
\hline Año residencia & $\mathrm{R} 2$ & $\mathrm{R} 3$ & $\mathrm{R} 4$ & $\mathrm{R} 5$ & \\
$\quad$ Valor medio & 2.74 & 2.20 & 2.29 & 2.62 & \\
$\quad$ Número adjuntos & $<20$ & $20-39$ & $>40$ & & \\
$\quad$ Valor medio & 2.32 & 2.34 & 2.68 & & \\
Residentes (año) & 1 & 2 & 3 & 4 & 5 \\
$\quad$ Valor medio & 2.44 & 2.32 & 2.38 & 3.0 & 2.4 \\
& & & & &
\end{tabular}




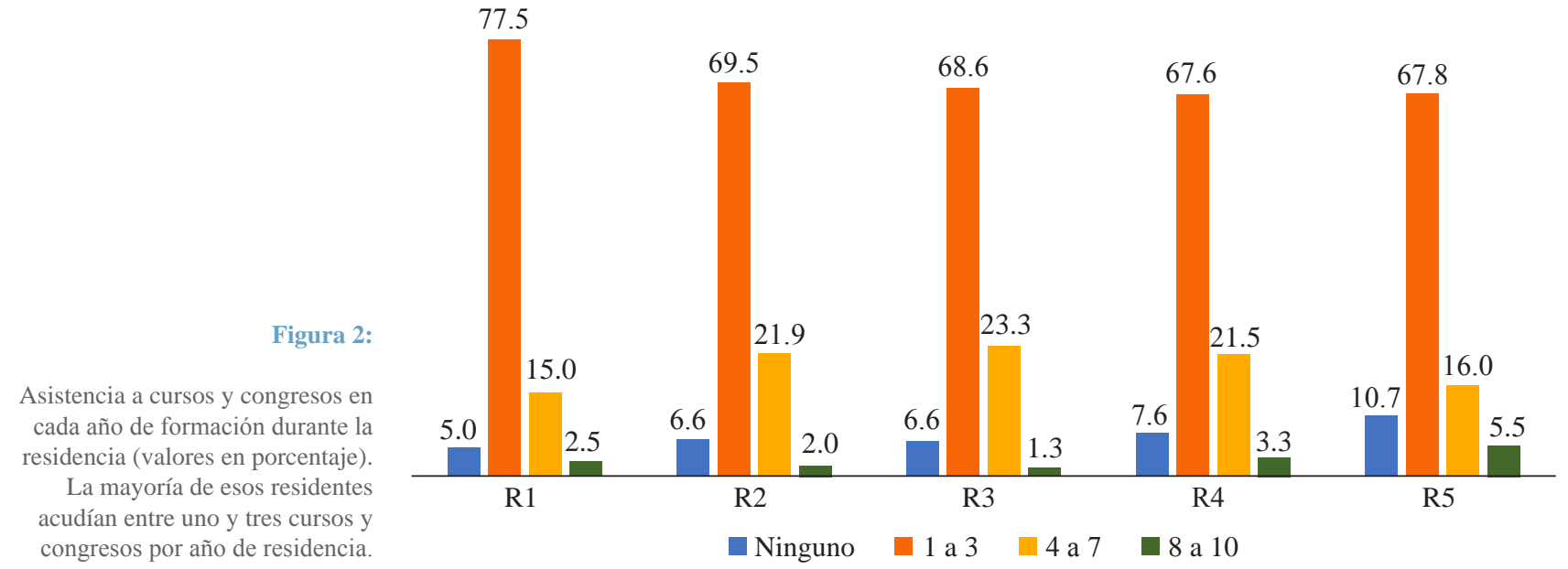

mente un tema para el desarrollo de la tesis, aunque sólo $9.2 \%$ indicaron haber iniciado la tesis doctoral. Los cursos, el tema a desarrollar y la tesis se iniciaban a partir del tercer año de residencia.

A futuro, un $65.8 \%$ de estos respondientes estarían interesados en realizar una rotación externa formativa en un centro nacional y $59.2 \%$ en un centro extranjero, siempre y cuando su programa individual de educación lo permitiera. La mayoría de ellos tenían preferencia por trabajar al finalizar la residencia en una Unidad de Muñeca y Mano en $18.3 \%$, seguido por la Medicina Deportiva en $14.1 \%$ y con menor interés en Raquis, Infantil y Sépticos (Figura 5). Las perspectivas laborales se consideraban como buenas a corto y medio plazo por $74.2 \%$ de los encuestados. Respecto a la influencia de la pandemia por coronavirus en la situación laboral actual, $21.7 \%$ de tales residentes respondieron que dicha influencia sería negativa.

\section{Discusión}

Los hallazgos más importantes del estudio fueron los siguientes: 1) $52.5 \%$ de los citados residentes no se sentían satisfechos respecto a su plan individual de formación; 2) $56.7 \%$ de ellos indicaron que su plan personal de entrenamiento no contemplaba la actividad científica; 3 ) no existía tiempo establecido con periodicidad semanal dedicado a labores de investigación; 4) sólo $35.8 \%$ de dichos entrevistados se sentían motivados por parte de sus adjuntos en torno a la gestión investigadora; 5) la asistencia a cursos y congresos y la presentación de comunicaciones estaba muy por encima de la publicación de trabajos científicos; 6) más de la mitad de tales encuestados estarían interesados en realizar una rotación externa formativa a nivel nacional o extranjera; 7) parece existir una mayor preferencia por la intervención de Muñeca y Mano y la Medicina Deportiva.

Estudios previos indican que la asignación de tiempo es el factor fundamental para incrementar la práctica científica. Al-Taha y colegas referían que $83 \%$ de los residentes de cirugía plástica en Canadá establecían la falta de tiempo como la principal barrera frente a las acciones de investigación. ${ }^{10}$ Levy y colaboradores publicaron que hubo un incremento de publicaciones entre los integrantes de la residencia de COT en USA de 12 al 18\% tras limitar el número de horas de trabajo semanales a $80 .{ }^{11}$ Williams y asociados referían, en su estudio de 1,690 miembros de residencia de COT en USA, un mayor volumen de publicaciones con programas de educación con tiempo dedicado a actividades de investigación. ${ }^{12}$

Torres y coautores compararon las publicaciones de 27 miembros de la residencia de Cirugía Ortopédica y Trauma-

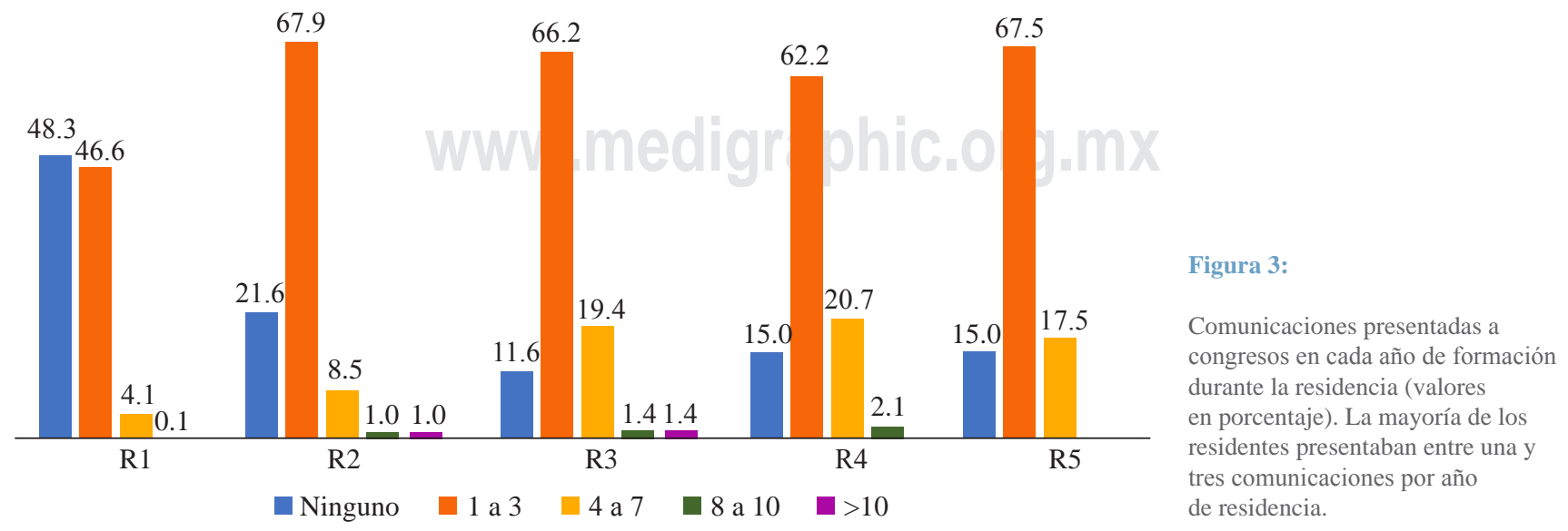




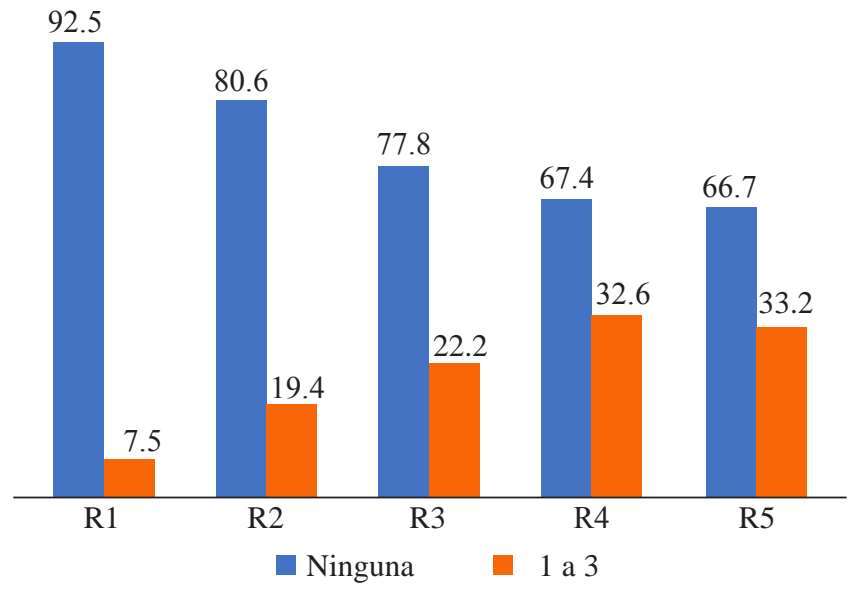

Figura 4: Publicaciones en revistas en cada año de formación durante la residencia (valores en porcentaje). El número de residentes con trabajos científicos se incrementaba conforme avanzaba el tiempo de residencia, aunque en un menor porcentaje que la asistencia a cursos y congresos o la presentación de comunicaciones.

tología en USA tras la instauración de un programa específico de tareas de investigación, con las publicaciones de 24 residentes antes de la aplicación del programa. Su conclusión fue que aquellos con programa específico tenían más publicaciones que otros sin programa, aunque el efecto no se mantenía tras terminar la residencia. ${ }^{8}$ También Voss y su grupo indicaron en su estudio de 129 integrantes de residencia de COT en Austria, Alemania y Suiza, una mayor actividad científica en los médicos con tiempo asignado a faenas de investigación. ${ }^{13}$ Sin embargo, otros estudios no encuentran un incremento de publicaciones en programas de formación con tiempo establecido para labores de investigación, aunque incluyen en su estudio las publicaciones de residentes y adjuntos. ${ }^{14,15}$ En el presente estudio, los médicos contestaron que su plan individual de entrenamiento no incluía aspectos relacionados ni asignaba tiempo a la práctica investigadora.

Otro aspecto importante por considerar es la motivación existente en el servicio hacia la labor investigativa. Parecería lógico que esos residentes de servicios con adjuntos investigadores tendrían una mayor práctica científica durante su período de entrenamiento. Voss y colegas indicaron que estos doctores dedicaban mayor tiempo consagrado a tareas de investigación, aunque sin encontrar diferencias significativas en relación con los médicos de servicios con menor ocupación investigadora por parte de sus adjuntos. ${ }^{13}$ En este estudio no se ha recogido la actividad investigadora de dichos adjuntos, pero sí la motivación de esos residentes por parte de los segundos, de tal modo, sólo uno de cada tres entrevistados respondieron que sí se sentían motivados por sus adjuntos para realizar gestión investigadora.

El interés de los galenos en la investigación científica también puede verse influenciado por la situación social e incluso por el cambio generacional. ${ }^{16}$ El desarrollo de la tecnología digital ha influido de manera directa en el comportamiento de las personas entre una generación y la siguiente.
Las generaciones de millenials y postmillenials a las cuales pertenecen estos doctores han tenido acceso a cualquier tipo de información al momento, no consideran el trabajo como su primera prioridad, aunque su nivel de compromiso es alto y cuestionarlo todo se ha convertido en algo natural para ellos. Estos aspectos deberían ser tenidos en cuenta al diseñar un programa de investigación.

Los sentimientos de insatisfacción son la fase inicial del síndrome de burnout. Ramírez y coautores refieren una prevalencia del síndrome de burnout de $29 \%$ en 119 doctores de diferentes especialidades de los hospitales del área sureste de Madrid. ${ }^{4}$ Sin embargo, Saavedra y colegas publican un porcentaje de $50 \%$ en residentes de medicina y sicología clínica del área sanitaria IV de Asturias. ${ }^{5}$ En este estudio, 52\% de aquellos interrogados indicaron valores por debajo de la media en la escala de valoración de satisfacción, sin relación con el año de residencia, las características del servicio (número de titulares y adjuntos) o tiempo invertido en jornada ordinaria o complementaria. Sí se encuentra correspondencia con la asignación de actividad asistencial tras la guardia, a diferencia del estudio de Ramírez y colaboradores. ${ }^{4}$

El presente estudio representa una muestra reducida del total de residentes de Cirugía Ortopédica y Traumatología en España, alrededor de $10 \%$ de los estudiantes quienes cursan la especialidad actualmente, lo cual es una limitación a tenerse en cuenta. El índice de respuestas puede estar influenciado por el método utilizado para contactar al residente (grupos de WhatsApp) o por el interés mismo del galeno en responder a la encuesta o bien por no haber realizado una mejor campaña de información ante tales residentes. Los resultados parecen indicar que los susodichos acceden fácilmente a cursos y congresos y a realizar comunicaciones. Sin embargo, dicha

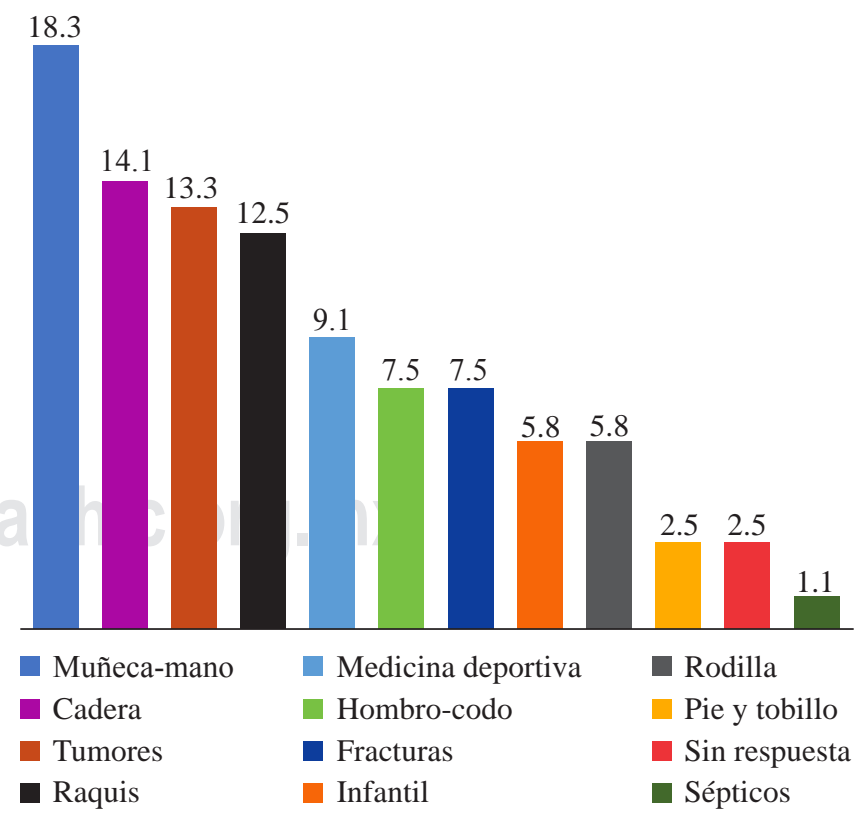

Figura 5: Campo de preferencia de los residentes al terminar el período de formación (valores en porcentaje). Existía una mayor preferencia por la Cirugía de Muñeca y Mano y la Medicina Deportiva. 
labor no se materializa en un conjunto similar de publicaciones. Por ello, se sugiere que se anime a estos profesionales a utilizar el trabajo realizado en la comunicación hacia la redacción de una publicación científica. También ha llamado la atención cómo sólo 9.2\% de aquellos encuestados habían iniciado el máster de formación e investigación.

La tesis doctoral permite realizar una investigación rigurosa y conseguir el nivel académico de doctor, lo cual amplía el campo de salidas profesionales académico-universitaria e institucionales. Esto sería también un aspecto por potenciar en los servicios de COT. En cuanto a la actividad asistencial, el número medio de horas trabajadas y la cuantía de guardias mensuales se ajustaba a lo establecido en el Real Decreto 1146/2006, del 06 de octubre, que regula la relación laboral especial de residencia para la formación de especialistas en Ciencias de la Salud y establece una duración máxima de la jornada laboral semanal de 37.5 horas y siete guardias al mes. Sin embargo, $63.5 \%$ indicaron tener asignada tarea asistencial tras la guardia y $45 \%$ acometía labor asistencial fuera de su período laboral en el hospital, aspectos totalmente incompatibles con la legislación actual. A futuro, existe un elevado interés en realizar rotaciones externas formativas específicas en centros nacionales y extranjeros, así como preferencias por unidades específicas, lo cual parece indicar una tendencia cada vez mayor hacia la subespecialización en una región anatómica.

En el conocimiento de los autores de este escrito, el presente estudio es el primero que se realiza entre residentes de COT en España. Por ello, los resultados divulgados podrían aplicarse para la mejora de los planes individuales de preparación en torno a las actividades de investigación y la tarea asistencial de tales médicos y con la meta de incrementar su nivel de satisfacción.

\section{Conclusiones}

Más de la mitad de aquellos residentes encuestados realizan labor asistencial el día siguiente a la guardia y prácticamente uno de cada dos de ellos atendían ocupaciones asistenciales fuera de su período laboral en el hospital.

Los médicos involucrados indicaron que su plan individual de educación no incluía aspectos relacionados con la actividad investigadora y que no se asignaba tiempo para dichas ocupaciones, en una proporción mayor de $50 \%$.

Un porcentaje no despreciable de médicos no estaban satisfechos con su programa de formación.

Referencias

1. Marugán de Miguelsanz JM, Eiros Bouza JM. Situación actual de la formación médica especializada en España. Educ Med. 2016; 17: 51-4.
2. Fernández PJ. Formación especializada en España: el internado rotatorio a la troncalidad. Educ Med. 2015; 16: 57-67.

3. Tutosaus-Gómez JD, Morán-Barrios J, Pérez-Iglesias F. Historia de la formación especializada en España y sus claves docentes. Educ Med. 2018; 19: 229-34.

4. Ramírez PMA, Garitano QLF, González AJM, González BEJ, Sánchez UMA, Del Campo BMT. Síndrome de burnout en médicos residentes de los hospitales del área sureste de la Comunidad de Madrid. Rev Asoc Esp Espec Med Trab. 2019; 28(1): 57-65.

5. Saavedra RI, García GJV, Llamazares GFJ, Arbesú FE, López DA. Grado de burnout en especialistas en formación de medicina y psicología clínica. Educ Med. 2021; 22(Suppl. 2): 55-61. Disponible en: https://doi.org/10.1016/j.edumed.2019.05.002.

6. Macknin JB, Brown A, Marcus RE. Does research participation make a difference in residency training? Clin Orthop Relat Res. 2014; 472: 370-6. doi: 10.1007/s11999-013-3233-y.

7. Ahn J, Donegan DJ, Lawrence JT, Halpern SD, Mehta S. The future of the orthopaedic clinician-scientist: part II: Identification of factors that may influence orthopaedic residents' intent to perform research. $J$ Bone Joint Surg Am. 2010; 92: 1041-6.

8. Torres D, Gugala Z, Lindsey RW. A dedicated research program increases the quantity and quality of orthopaedic resident publications. Clin Orthop Relat Res. 2015; 473(4): 1515-21. doi: 10.1007/s11999014-4080-1.

9. Namdari S, Jani S, Baldwin K, Mehta S. What is the relationship between number of publications during orthopaedic residency and selection of an academic career? J Bone Joint Surg Am. 2013; 95(7): e45.

10. Canadian Plastic Surgery Research Collaborative (CPSRC). Barriers and attitudes to research among residents in plastic and reconstructive surgery: a national multicenter cross-sectional study. J Surg Educ. 2017; 74(6): 1094-104. doi: 10.1016/j.jsurg.2017.04.004.

11. Levy DM, Luchetti TJ, Levine BR. Have residents produced more research since the inception of the 80-hour workweek? Iowa Orthop J. 2017; 37: 205-9.

12. Williams BR, Agel JA, Van Heest AE. Protected time for research during orthopaedic residency correlates with an increased number of resident publications. J Bone Joint Surg Am. 2017; 99(13): e73. doi: 10.2106/JBJS.16.00983.

13. Voss A, Andreb B, Pauzenberger L, Hersbt E, Pogorzelski J, John $\mathrm{D}$, et al. Research productivity during orthopedic surgery residency correlates with pre-planned and protected research time: a survey of German-speaking countries. Knee Surg Sports Traumatol Arthrosc. 2021; 29: 292-9. doi: 10.1007/s00167-020-05983-w.

14. Krueger CA, Hoffman JD, Balazs GC, Johnson AE, Potter BK, Belmont PJ Jr. Protected resident research time does not increase the quantity or quality of residency program research publications: a comparison of 3 orthopedic residencies. J Surg Educ. 2017; 74(9): 264-70.

15. Osborn PM, Ames SE, Turner NS, et al. An analysis of research quality and productivity at six academic orthopaedic residencies. $J$ Surg Educ. 2018; 75(6): 1635-42. doi: 10.1016/j.jsurg.2018.04.022.

16. Seil R. Why ESSKA? On what it takes for orthopaedic surgeons and their scientific societies to adapt to societal changes in 2018. Knee Surg Sports Traumatol Arthrosc. 2019; 27: 665-72. doi: 10.1007/ s00167-019-05371-z.

Conflictos de intereses: Los autores del presente estudio declaran no tener ningún conflicto de intereses.

Financiación: La presente investigación no ha recibido ayudas específicas provenientes de agencias del sector público, sector comercial ni entidades sin ánimo de lucro. 
Anexo 1: Cuestionario anónimo de valoración de la actividad investigadora y científica y el grado de satisfacción de los residentes de Cirugía Ortopédica y Traumatología.

1. Edad

2. Sexo
a. Varón
b. Mujer

3. Financiación hospital
a. Pública
b. Pública gestión privada
c. Privada

4. Provincia

5. Número de adjuntos en el servicio (incluyendo jefe de servicio y jefes de sección)

6. Número de residentes en el servicio

7. Horas semanales de trabajo (sin guardias)

8. Número de guardias al mes (sin contar período vacacional)

9. Indica tu año de residencia
a. $\mathrm{R} 1$
b. R2
c. R3
d. $\mathrm{R} 4$
e. R5

10. ¿Tu plan individual de formación contempla como objetivo la actividad científica ajustada a cada año de residencia?

a. Sí

b. No

11. Asistencia a cursos y congresos con (número) durante la residencia
a. $\mathrm{R} 1$
b. R2
c. R3
d. R4
e. $\mathrm{R} 5$

12. Comunicaciones presentadas a congresos (número) durante la residencia
a. R1
b. R2

c. R3

d. R4

e. $\mathrm{R} 5$

13. Publicaciones (número) durante la residencia
a. R1
b. R2
c. R3
d. R4
e. R5

14. ¿Has iniciado el máster de formación e investigación?
a. Sí
b. No

15. ¿Has iniciado la tesis doctoral?

a. Sí

b. No

16. ¿Tienes en mente un tema para desarrollo como tesis doctoral?
a. Sí
b. No

17. ¿Tienes tiempo asignado semanalmente para actividades de investigación?
a. Sí
b. No

18. Si has contestado afirmativamente a la pregunta anterior, ¿cuántas horas semanales tienes asignadas?

19. ¿Consideras que es tiempo suficiente para actividades de investigación?
a. Sí
b. No

20. ¿Cuánto tiempo querías dedicar semanalmente a tareas de investigación?

21. ¿Consideras que existe una motivación de tus adjuntos para la actividad científica en los residentes?
a. Sí
b. No 
22. Indica el nivel de satisfacción con respecto a la formación en la actividad científica en tu servicio de los residentes

Escala de 1 a 5

$1=$ No estoy satisfecho

$5=$ Estoy muy satisfecho

23. ¿Has realizado o planeas realizar una rotación externa formativa específica en algún centro extranjero?
a. Sí
b. No

24. ¿Has realizado o planeas realizar una rotación externa formativa específica en algún centro nacional?
a. Sí
b. No

25. ¿'Tienes actividad asistencial asignada al día siguiente de una guardia? Calcula el porcentaje según el número de horas de una jornada habitual.
a. No
b. Menos de un $10 \%$
c. Más del $50 \%$
d. Más del $80 \%$
e. Como una jornada habitual

26. ¿Realizas alguna actividad asistencial extra fuera de horario laboral?
a. Sí
b. No

27. ¿Tienes algún campo en que te gustaría dedicar tu futuro al acabar la residencia?
a. Cirugía de pie y tobillo
b. Cirugía de mano
c. Cirugía de rodilla
d. Cirugía de cadera
e. Cirugía de columna
f. Cirugía de hombro/codo
g. Cirugía tumoral
h. Ortopedia infantil
i. Medicina deportiva/cirugía artroscópica
j. Otros

28. ¿Consideras buenas las perspectivas laborales al terminar la residencia a corto y medio plazo?
a. Sí
b. No

29. ¿Piensas que tendrás alguna dificultad para encontrar trabajo al terminar la residencia?
a. Sí
b. No

30. ¿Piensas que la situación laboral ha empeorado con la crisis de coronavirus?

a. Sí

b. No 